\title{
Isolated Potentials and Pace-Mapping as Guides for Ablation of Ventricular Tachycardia in Various Types of Nonischemic Cardiomyopathy
}

\author{
MICHAEL KÜHNE, M.D., GERALD ABRAMS, M.D., JEAN-FRANCOIS SARRAZIN, M.D., \\ THOMAS CRAWFORD, M.D., ERIC GOOD, D.O., AMAN CHUGH, M.D., \\ MATTHEW EBINGER, D.O., KRIT JONGNARANGSIN, M.D., FRANK PELOSI, JR., M.D., \\ HAKAN ORAL, M.D., FRED MORADY, M.D., and FRANK M. BOGUN, M.D.
}

From the University of Michigan Medical Center, Ann Arbor, Michigan, USA

Nonischemic Cardiomyopathy and Ventricular Tachycardia. Background: In patients with prior infarction, isolated potentials (IPs) during sinus rhythm reflect fixed scar and often indicate sites critical for ventricular tachycardia (VT). The purpose of this study was to determine the value of IPs in conjunction with pace-mapping to guide VT ablation in patients with various types of nonischemic cardiomyopathy.

Methods: Mapping and ablation of VT were performed in 35 consecutive patients (26 men, age $55 \pm$ 13 years, ejection fraction $0.31 \pm 0.14$ ) with $\mathrm{VT}$ and various etiologies of nonischemic cardiomyopathy. Pace-mapping was performed at sites with low voltage. Radiofrequency energy was delivered at sites with concealed entrainment or matching pace-maps.

Results: One hundred ninety-five VTs (mean cycle length $363 \pm 88 \mathrm{~ms}$ ) were induced. Sites with prespecified ablation criteria displaying IPs during sinus rhythm were recorded in 21 of 35 patients $(60 \%$, IP-positive). In these patients, a total of 216 sites meeting prespecified ablation criteria were identified and 146 of 216 sites (68\%) displayed IPs. Fifteen of 21 IP-positive patients (71\%) no longer had inducible VT after ablation. In 14 of 35 patients, no sites with IPs where prespecified ablation criteria were met were identified (IP-negative) despite combined endocardial and epicardial mapping in 7 of 14 patients. Only 1 of 14 IP-negative patients (7\%) no longer had inducible VT at the end of the ablation procedure. During a mean follow-up of $18 \pm 13$ months, 14 of 21 IP-positive patients $(67 \%)$ remained arrhythmia-free, compared to 1 of 14 IP-negative patients $(7 \%$; $P<0.01)$. Half of the IP-negative patients had major adverse events due to recurrent arrhythmias, compared to none in IP-positive patients.

Conclusion: IPs in conjunction with pace-mapping are helpful for identifying critical isthmus areas for ablation of VT in patients with various types of nonischemic cardiomyopathy. Patients with nonischemic cardiomyopathy in whom the arrhythmogenic substrate is characterized by IPs have a more favorable outcome than patients in whom IPs are absent. (J Cardiovasc Electrophysiol, Vol. 21, pp. 1017-1023, September 2010)

arrhythmogenic right ventricular dysplasia, cardiomyopathy, radiofrequency ablation, sarcoid, ventricular tachycardia

\section{Introduction}

Isolated potentials (IPs) during sinus rhythm indicate areas of fixed scar in patients with previous myocardial infarction. ${ }^{1}$ In addition to pace-mapping, which has been used to identify exit sites of reentry circuits, ${ }^{2}$ IPs have been found to be helpful in guiding radiofrequency catheter ablation of ventricular tachycardia (VT) in postinfarction patients. ${ }^{3}$ Localized areas of myocardial fibrosis resulting in electrogram fragmentation are frequently found and may represent the

H. Oral reports research grants supported by St. Jude Medical and Boston Scientific. Other authors: No disclosures.

Address for correspondence: Frank Bogun, M.D., Division of Cardiology, University of Michigan Health System, 3119 TC, 1500 E. Medical Center Dr., Ann Arbor, MI 48109-0366, USA. Fax: 734-936-7026; E-mail: fbogun@med.umich.edu

Manuscript received 1 August 2009; Revised manuscript received 18 January 2010; Accepted for publication 1 February 2010.

doi: 10.1111/j.1540-8167.2010.01756.x anatomic substrate for VT in patients with nonischemic cardiomyopathy. ${ }^{4}$

The purpose of this study was to assess the value of IPs during sinus rhythm in conjunction with pace-mapping for identifying potential target sites for VT ablation in patients with various types of nonischemic cardiomyopathy.

\section{Methods}

\section{Patient Characteristics (Table 1)}

The subjects of this study were 35 consecutive patients (26 males, age $55 \pm 13$ years, ejection fraction $0.31 \pm 0.14$ [mean \pm 1 standard deviation]) with nonischemic cardiomyopathy who were referred for mapping and ablation of VT. In 30 patients, the indication for VT ablation was recurrent implantable cardioverter-defibrillator (ICD) shocks. Seven patients $(20 \%)$ had cardiac sarcoidosis, 4 patients had arrhythmogenic right ventricular dysplasia (ARVD) (11\%), and the remainder had idiopathic dilated cardiomyopathy (69\%). Twelve-lead ECGs of the clinical VT were available in 16 of 35 patients. All patients had failed antiarrhythmic 


\section{TABLE 1}

Patient Characteristics

\begin{tabular}{lc}
\hline Total number of patients & 35 \\
Age (years) & $55 \pm 13$ \\
Male (no. \%) & $26(74 \%)$ \\
LVEF (\%) & $31 \pm 13$ \\
Idiopathic DCM (\%) & $24(68 \%)$ \\
Sarcoid heart disease (\%) & $7(21 \%)$ \\
ARVD (\%) & $4(11 \%)$ \\
\hline
\end{tabular}

ARVD $=$ arrhythmogenic right ventricular dysplasia; $\mathrm{DCM}=$ dilated cardiomyopathy; $\mathrm{LVEF}=$ left ventricular ejection fraction.

treatment including amiodarone in 14 patients. Thirty patients $(86 \%)$ had an ICD. Three patients underwent ICD implantation after the ablation procedure. Two patients refused ICD implantation.

A total of 195 VTs were inducible by programmed ventricular stimulation. The mean VT cycle length was $363 \pm$ $88 \mathrm{~ms}$. Ninety-six of the VTs (49\%) had a left bundle branch block morphology and 99 (51\%) had a right bundle branch block morphology. None of the VTs was due to bundle branch reentry.

\section{Electrophysiologic Study}

This study was approved by the University of Michigan Institutional Review Board. After informed consent was obtained, vascular access was obtained in a femoral vein. Two multielectrode catheters were positioned in the His position and the right ventricular apex. Programmed ventricular stimulation from at least 2 right ventricular sites was performed using up to 4 extrastimuli before and after the ablation procedure. ${ }^{5}$ Three thousand units of heparin was administered followed by an additional 1,000 units per hour for right-sided procedures. For left ventricular procedures an activated clotting time of $>300$ seconds was targeted.

For mapping and ablation an 8 French open irrigatedtip catheter with a 3.5 -mm-tip electrode $\left(\right.$ Thermocool ${ }^{\circledR}$; Biosense Webster, Diamond Bar, CA, USA) was used in conjunction with a 3-dimensional electroanatomic mapping system (CARTO; Biosense Webster). In 8 patients, a 4-mmtip ablation catheter (Navistar; Biosense Webster) was used for mapping and ablation. Intracardiac electrograms and surface electrocardiograms were displayed on an oscilloscope and recorded on optical disc (EP Med Systems Inc., West Berlin, NJ, USA).

\section{Mapping Procedure}

Voltage maps were generated during sinus rhythm (Fig. 1). An IP was defined as a distinct potential separated from the ventricular electrogram by an isoelectric segment, and/or a segment with low amplitude noise $(<0.05 \mathrm{mV})$ of $>20 \mathrm{~ms}$ duration at a gain of $40-80 \mathrm{~mm} / \mathrm{mV}$ (Fig. 2). Pacemapping was performed at sites with low voltage $(<1.5 \mathrm{mV}$; Fig. 2). Electrograms were assessed by 2 independent observers. Discrepancies were resolved by consensus. Representative examples of IPs are shown in Figure 3.

A matching pace-map was defined as a pace-map in which $\geq 10 / 12$ leads matched with the QRS morphology of the induced VT. ${ }^{3}$

Hemodynamically tolerated VTs were mapped by entrainment mapping. An isthmus site was defined as a site demon-

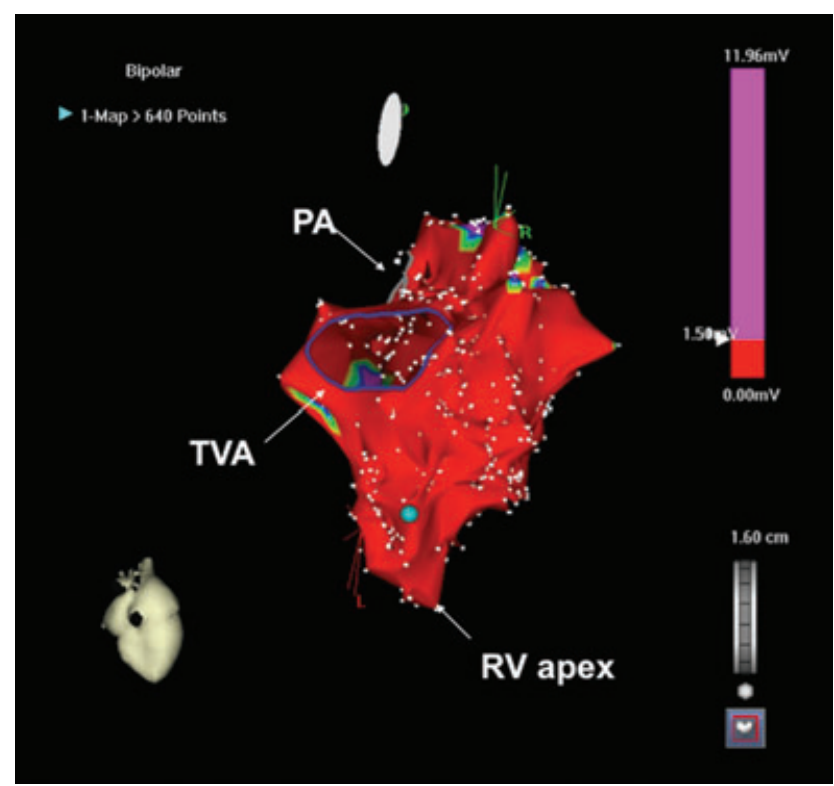

Figure 1. Electroanatomic bipolar voltage map of the right ventricle in a patient with nonischemic cardiomyopathy (right anterior oblique view with caudal tilt). Pulmonary artery (PA), tricuspid valve annulus (TVA), and right ventricular apex are indicated. Large areas with low voltage are present. The blue tag denotes the site of an isolated potential (IP) during sinus rhythm (Fig. 2) and a matching pace-map for one of the patient's VTs (Fig. 3). The site was an effective ablation site for this VT.

strating concealed entrainment with matching stimulus-QRS and electrogram-QRS intervals and VT termination during ablation.

Pace-mapping was performed for nontolerated VTs at the cycle length of the targeted VT. If multiple VTs were targeted, pacing was performed at the mean cycle length of the targeted VTs.

If multiple VTs were targeted, pacing was performed with a cycle length averaging the targeted VTs. An isthmus area
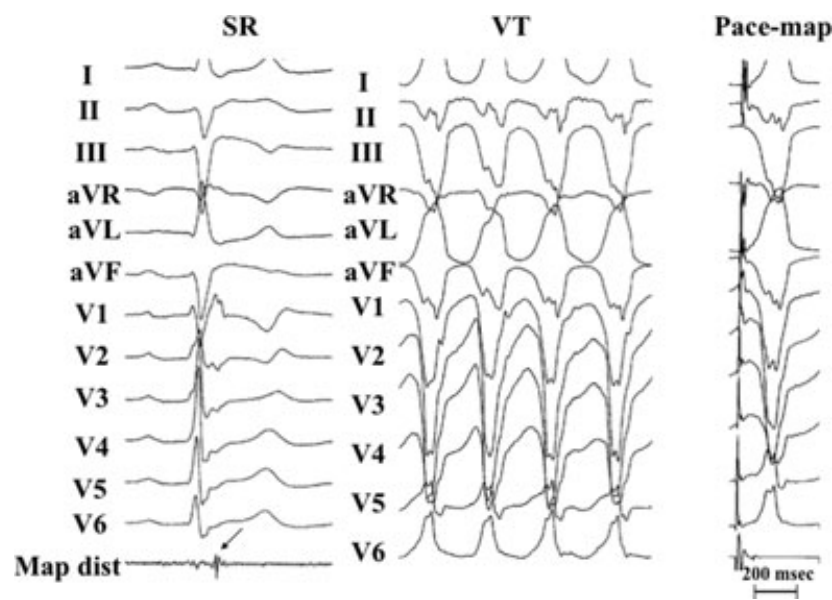

Figure 2. Left panel: An IP (arrow) recorded with the distal electrodes of the mapping catheter (Map dist) at the right ventricular site shown in Figure 1. The IP has an amplitude of $0.23 \mathrm{mV}$ and is separated from the ventricular electrogram by $50 \mathrm{~ms}$. Middle panel: Twelve-lead ECG showing $V T$ at a cycle length of $280 \mathrm{~ms}$, with a left bundle branch block morphology and superior axis (left). Right panel: Pacing from the right ventricular site shown in Figure 1 resulted in a matching pace-map (right). 
A

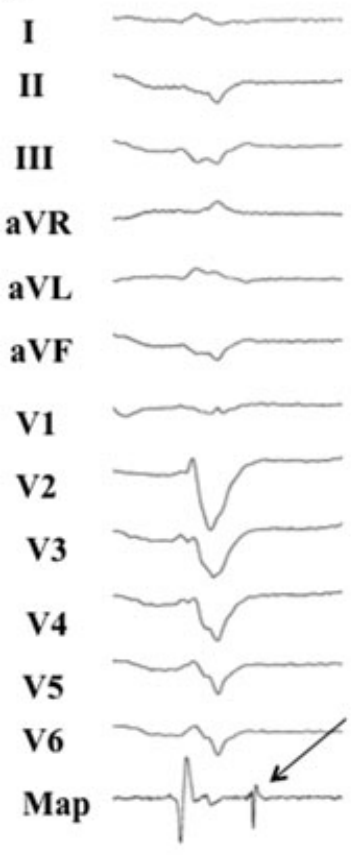

B

\section{I}

II

III

aVR

aVL

aVF

v1

v2

v3

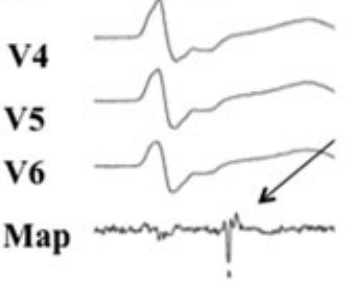

C

$$
\text { I }
$$

II

III

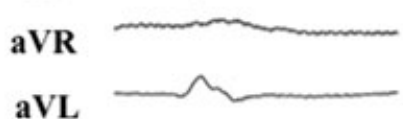

aVL

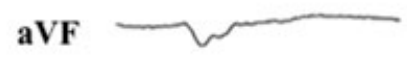

v1

v2

v3

$\mathrm{V} 4$

v5

V6

Map

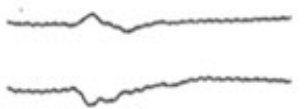

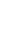

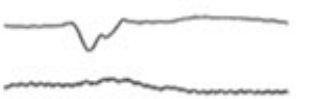

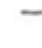
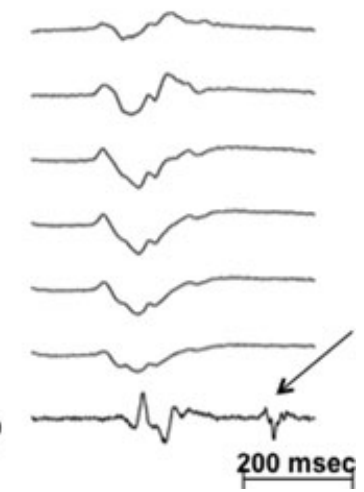

Figure 3. IPs (arrows) recorded by the mapping catheter (Map) in various types of cardiomyopathy. Panel A shows IPs in a patient with dilated cardiomyopathy, panel B shows an example of IPs in a patient with cardiac sarcoidosis, and panel C shows IPs in a patient with arrhythmogenic right ventricular cardiomyopathy.

was defined as an area where there was a matching pace-map and noninducibility of the targeted VT after the ablation. The prespecified criteria for delivery of radiofrequency energy were the presence of concealed entrainment (Fig. 4) or the presence of matching pace-maps. IPs were noted but were not by themselves a criterion to deliver radiofrequency energy.

\section{Radiofrequency Ablation}

Radiofrequency energy was delivered if prespecified criteria were present. All inducible VTs were targeted. If these criteria were present in the vicinity of an anatomical barrier (tricuspid, mitral, pulmonary, or aortic annulus), the ablation lesion was continued to the annulus as long as this region displayed low voltage. Otherwise, ablation was delivered at sites with matching pace-maps.

The endpoint of the procedure was noninducibility of all inducible VTs for which an isthmus was identified. Pro-

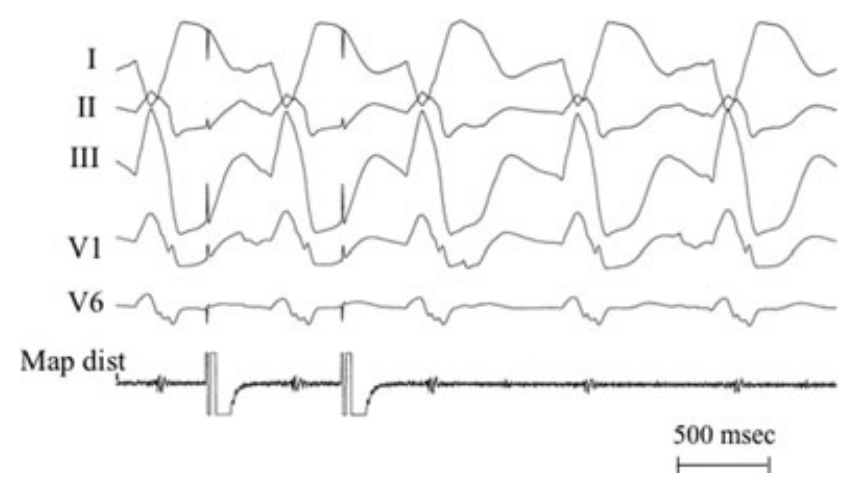

Figure 4. Concealed entrainment in a patient with nonischemic cardiomyopathy. grammed stimulation was repeated after the ablation procedure from 2 right ventricular sites with up to 4 extrastimuli.

Radiofrequency ablation was performed using a Stockert RF generator (Biosense Webster). Radiofrequency energy was delivered for 60-120 seconds at an initial output of $35 \mathrm{~W}$ and/or until the capture threshold post-ablation was $>10 \mathrm{~mA}$. Power was titrated to achieve a $10 \mathrm{ohms}$ impedance drop; maximal temperature was set to $45^{\circ} \mathrm{C}$ for irrigated tip catheters. For nonirrigated catheters, the target temperature was set to $60^{\circ} \mathrm{C}$ at a power of $30-70 \mathrm{~W}$.

\section{Data Analysis}

Patients in whom IPs were recorded during electroanatomic mapping (IP-positive) were compared to patients in whom no IPs were recorded (IP-negative). In 7 of 14 patients in whom no IPs were recorded during endocardial mapping, transcutaneous epicardial mapping $(n=4)$ or limited epicardial mapping through the coronary venous system $(\mathrm{n}=3)$ also failed to identify sites displaying IPs. A mean of $356 \pm 237$ points/patient were registered during the baseline rhythm (331 \pm 222 points in IP-positive patients compared to $392 \pm 262$ points in IP-negative patients; $P=0.5$ ). Measurements of electrogram amplitude and width were performed with electronic calipers. If an IP was present, the total width of the electrogram was measured from the onset of the electrogram to the end of the IP. Also the interval from the end of the QRS complex to the IP was measured.

\section{Histology}

In 2 patients in whom no IPs were recorded during left and right ventricular endocardial and epicardial mapping, cardiac transplantation was performed for uncontrolled VT 14 and 26 days after the ablation procedure. Samples of myocardium 
were procured from the septum, anterior wall, and lateral wall of the left ventricle, midway between the apex and base. Tissue samples also were procured from ablation sites and sites immediately adjacent to the ablation lesions. The tissue sections were stained with hematoxylin/eosin and trichrome stains.

\section{Statistical Analysis}

Continuous variables are expressed as mean \pm 1 standard deviation. Comparisons were performed using Student's $t$ test. Discrete variables were compared using chi-square test or the Fisher exact test, as appropriate. A P-value of $<0.05$ indicated statistical significance.

\section{Follow-up}

The patients were followed every 3-6 months in the ICD clinic to assess for VT recurrences. Major adverse events were defined as death, implantation of a left ventricular assist device, or cardiac transplantation.

\section{Results}

One hundred ninety-five VTs (mean cycle length $363 \pm$ $88 \mathrm{~ms}$ ) were induced. None of the VTs were due to bundle branch reentry. A total of 882 distinct sites where pacemapping was performed were analyzed. The mean electrogram amplitude at these sites was $0.67 \pm 0.77 \mathrm{mV}$, with a mean width of $139 \pm 96 \mathrm{~ms}$. IPs were identified in 21 of 35 patients (IP-positive) at 213 of 882 sites (24\%). In $39 \%$ of the sites with IPs, the electrogram extended beyond the end of the QRS complex. IP-positive patients had a mean of $5.3 \pm 5.2$ (range 1-22) inducible VTs. In 14 of 35 patients (IP-negative), no IPs were identified despite extensive mapping, including epicardial mapping in 7 of the 14 patients. IP-negative patients had a mean of $6.0 \pm 4.5$ (range: 1-13) inducible VTs.

\section{Ablation Sites}

All inducible VTs were targeted for ablation. Forty-two of the VTs were hemodynamically tolerated and 17 VTs were mapped with entrainment mapping. Three hundred thirteen sites meeting the prespecified ablation criteria were identified for 84/195 VTs (43\%). After ablation, 144 of 195 VTs (74\%) were no longer inducible. Sixteen of 35 patients (46\%) no longer had inducible VT at the end of the ablation procedure. Sites meeting prespecified ablation criteria were identified by pace-mapping in 73 of 84 VTs and by entrainment mapping in 11 of 84 VTs. Acute efficacy was similar when an irrigated tip catheter was used $(n=27)$ compared to when a nonirrigated catheter $(\mathrm{n}=8)$ was used $(\mathrm{P}=0.7)$.

\section{Isolated Potentials and Pace-Mapping (Table 2)}

IPs were present more frequently at sites with matching pace-maps than at sites with inadequate pace-maps $(\mathrm{P}<$ 0.0001). Sixty-nine percent of the sites displaying IPs had matching pace-maps; on the other hand, pacing at sites with low voltage alone, in the absence of IPs, less often showed matching pace-maps $(26 \%$; $\mathrm{P}<0.0001)$. The mean electrogram width and the stimulus-QRS interval were longer and the electrogram amplitude was smaller at sites with IPs compared to sites where IPs were absent. IPs extended $124 \pm$ $133 \mathrm{~ms}$ beyond the end of the QRS complex. The presence

\section{TABLE 2}

Comparison of Sites With and Without Isolated Potentials

\begin{tabular}{llll}
\hline & IP Present & IP Absent & P-Value \\
\hline No. of evaluated sites & 213 & 669 & - \\
Amplitude (mV) & $0.45 \pm 0.71$ & $0.74 \pm 0.77$ & $<0.0001$ \\
Electrogram width (ms) & $254 \pm 136$ & $103 \pm 28$ & $<0.0001$ \\
S-QRS (ms) & $91 \pm 44$ & $48 \pm 21$ & $<0.0001$ \\
Matching pace-map (\%) & $146 / 213(69 \%)$ & $175 / 669(26 \%)$ & $<0.0001$ \\
\hline IP = isolated potentials; S-QRS = stimulus-QRS interval.
\end{tabular}

of IPs during VT was associated with the presence of IPs. In 7 of $21 \mathrm{IP}+$ patients, a total of 10 sites were assessed during baseline rhythm and during VT and 9 of 10 sites had IPs during VT and during baseline rhythm.

\section{Ablation and Relation to Isolated Potentials (Tables 2 and 3)}

In 21 of 35 patients $(60 \%)$, IPs were present at sites where ablation criteria were met (IP-positive patients). In this group, 111 VTs were inducible (5.3 \pm 5.2 VTs per patient). At least 1 site where the prespecified ablation criteria were met was identified in each of the 21 patients. Table 3 compares electrophysiologic characteristics in patients with and without IPs. In 1 of 21 patients, no site with IPs and no site where ablation criteria were met could be identified endocardially; transcutaneous epicardial mapping demonstrated 3 distinct epicardial sites with IPs and all 3 VTs were eliminated by ablation. In 2 other patients, limited epicardial mapping through the coronary venous system revealed areas with IPs that also were critical to the VT in both patients. Both patients had a single inducible VT that was effectively ablated within the great cardiac vein.

In the 21 IP-positive patients, prespecified ablation criteria were met at 216 sites for 62 of 111 VTs (56\%); 146 of 216 sites $(68 \%)$ displayed IPs. After ablation, 94 of $111 \mathrm{VTs}$ $(85 \%)$ were no longer inducible. A total of 15 of 21 IPpositive patients $(71 \%)$ with IPs had no inducible VT at

\section{TABLE 3}

Characteristics of Patients With and Without Isolated Potentials

\begin{tabular}{lccc}
\hline & $\begin{array}{c}\text { IP-Positive } \\
\text { Patients } \\
(\mathbf{n = 2 1 )}\end{array}$ & $\begin{array}{c}\text { IP-Negative } \\
\text { Patients } \\
(\mathbf{n = 1 4 )}\end{array}$ & P-value \\
\hline Points sampled per patient & $331 \pm 222$ & $392 \pm 262$ & 0.48 \\
Paced sites (total) & 449 & 433 & \\
Induced VT morphology & $55 / 56$ & $41 / 43$ & 0.9 \\
$\quad$ (LBBB/RBBB) & 11 & 13 & 0.02 \\
Idiopathic DCM (n) & 6 & 1 & 0.2 \\
Sarcoid heart disease (n) & 4 & 0 & 0.13 \\
ARVD (n) & $5.3 \pm 5.2$ & $6.0 \pm 4.5$ & 0.68 \\
Number of VTs & $0.64 \pm 0.86$ & $0.70 \pm 0.66$ & 0.17 \\
Amplitude (mV) & $174 \pm 122$ & $103 \pm 27$ & $<0.0001$ \\
Electrogram width (ms) & $69 \pm 40$ & $47 \pm 20$ & $<0.0001$ \\
S-QRS (ms) & $0.18 \pm 0.23$ & - & - \\
IP amplitude (mV) & $111 \pm 111$ & - & - \\
IP separation (ms) & $216 / 449(48 \%)$ & $97 / 433(22 \%)$ & $<0.0001$ \\
Matching pace-map (\%) & &
\end{tabular}

ARVD = arrhythmogenic right ventricular dysplasia; $\mathrm{DCM}=$ dilated cardiomyopathy; $\mathrm{LBBB}=$ left bundle branch block morphology; RBBB = right bundle branch block morphology; $\mathrm{VT}=$ ventricular tachycardia. 
the end of the procedure. All VTs with a matching pacemap were no longer inducible after ablation. The presence of IPs was associated with successful ablation even when the patients with ARVD or sarcoidosis were excluded $(\mathrm{P}<$ 0.0001 ).

In the 14 IP-negative patients, $84 \mathrm{VTs}$ were inducible $(6.0 \pm 4.5$ VTs per patient). Four of these patients underwent transcutaneous epicardial mapping because no sites where ablation criteria were met could be identified endocardially; in 3 patients, the coronary venous system was mapped and the effective ablation site was identified in the great cardiac vein in one of the 3 patients. No IPs were recorded by epicardial mapping in any of the 7 patients (50\% of IP-negative patients) who underwent epicardal mapping. Fifteen epicardial sites met prespecified ablation criteria and resulted in ablation of 5 VTs. Despite extensive mapping including right ventricular, left ventricular and transcutaneous epicardial mapping, no IPs were recorded in the 4 patients who had a combined endocardial and epicardial approach. In IP-negative patients, a transcutaneous epicardial approach was used more often $(\mathrm{n}=4)$ compared to IP-positive patients $(\mathrm{n}=1 ; \mathrm{P}=0.1)$.

In the 14 IP-negative patients, 97 sites where prespecified ablation criteria were met were identified for 26 of 84 induced VTs $(31 \%)$. Despite matching pace-maps, only 21 of $26 \mathrm{VTs}$ were effectively ablated. Only 1 of 14 patients $(7 \%)$ had no inducible VT at the end of the ablation procedure.

Linear ablation lesions were part of the ablation lesions in 12 patients due to the proximity to an annulus of the sites where ablation criteria were met. The presence or absence of a linear lesion was not associated with the inducibility of VT after ablation (5 of 12 patients with a linear lesion had inducible VT at the end of the procedure, whereas 7 of 12 patients with a linear lesion had an acutely successful outcome; $\mathrm{P}=0.4$ ).

The mean procedure time was $375 \pm 100$ minutes (IPpositive patients: $395 \pm 122$ minutes, IP-negative patients $349 \pm 57$ minutes; $\mathrm{P}=0.2$ ), and the mean fluoroscopy time was $64 \pm 45$ minutes (IP-positive patients: $64 \pm 26$ minutes, IP-negative patients: $64 \pm 23$ minutes; $P=0.99)$. A mean of $41 \pm 32$ minutes of radiofrequency energy were delivered per patient (IP-positive patients: $50 \pm 37$ minutes, IP-negative $30 \pm 21$ minutes; $\mathrm{P}=0.06$ )

\section{Anatomic Distribution of Isolated Potentials and Assessment of Low-Voltage Areas}

IPs were located in low-voltage areas. The majority $(159 / 213$ sites $)$ of IPs were located in a low-voltage area $<0.5 \mathrm{mV}$; the remaining 54 sites were in a low voltage area of $0.5-1.5 \mathrm{mV}$. The endocardial low-voltage area in IP-positive patients was $64 \pm 52 \mathrm{~cm}^{2}$; in IP-negative patients, it was $71 \pm$ $59 \mathrm{~cm}^{2}(\mathrm{P}=0.8)$. In patients with epicardial procedures, the area of low voltage was greater in the epicardium than in the endocardium $\left(67 \pm 52 \mathrm{~cm}^{2}\right.$ compared to $160 \pm 49 \mathrm{~cm}^{2}$; $\mathrm{P}=0.02$ ). The low-voltage area was periannular in 16 of 21 IP-positive patients and in 12 of 14 IP-negative patients $(\mathrm{P}=0.9)$. Additional areas of low voltage that were not continuous with periannular low-voltage areas were present in 14 of 21 IP-positive and 12 of 14 IP-negative patients $(\mathrm{P}=$ $0.2)$. The IPs were right ventricular, including the peritricuspid area and the right ventricular outflow tract, in all patients with ARVD. In patients with cardiac sarcoidosis with VTs originating in the right ventricle, IPs were located in the per- itricuspid area in all 7 patients and in the right ventricular outflow tract in 3 of 7 patients. In patients with dilated cardiomyopathy, IPs were located in the periannular area in 11 of 24 patients. In 1 of 5 patients in whom both endocardial and epicardial mapping was performed, IPs were identified in the epicardial lateral LV free wall.

\section{Histology (Fig. 5)}

The left ventricular tissue samples showed a diffuse pattern of fibrosis. There was widespread fibrosis characterized by patches of fibrotic tissue, frequently anastomosing with fine extension from other patches, and surrounding bundles of myocytes. This pattern often merged into areas of diffuse fibrosis. These same patterns of fibrosis were present immediately adjacent to sites of pace-guided ablation. Despite the widespread interstitial fibrosis, the overall myocyte fiber orientation was uninterrupted. Ablation sites were necrotic, with organization along their periphery. The histologic findings were similar in both patients in whom samples were obtained.

\section{Follow-Up}

The mean follow-up duration was $18 \pm 13$ months. There was no difference in mortality between the 2 groups. Major adverse events occurred more frequently in IP-negative patients $(\mathrm{n}=7: 50 \%)$ than in IP-positive patients $(\mathrm{n}=2: 10 \%$; $\mathrm{P}=0.01)$. All events except one were related to VT/VF in IP-negative patients: VT resulting in death $(\mathrm{n}=1)$ and VT storm $(\mathrm{n}=4)$, and VF $(\mathrm{n}=1)$ resulting in placement of left ventricular assist device or heart transplantation $(\mathrm{n}=$ 5). One patient died from heart failure 4 days after an ablation procedure. This patient required intravenous inotropic agents before the ablation procedure and continued to require inotropic agents after the ablation procedure. One death occurred in the IP-positive patients 17 months after an effective ablation procedure due to heart failure; this patient did not have any VT after ablation. One other IP-positive patient underwent cardiac transplantation for worsening heart failure. No VT-related adverse events occurred in the IP-positive patients. No other adverse events occurred during or after the ablation procedure.

During follow-up, treatment with antiarrhythmic medications was continued in 11 of 21 IP-positive patients. The remaining 10 IP-positive patients were not treated with antiarrhythmic medications during follow-up. Treatment with antiarrhythmic medications was continued in all but 2 IPnegative patients, and in 3 of these patients, another antiarrhythmic medication was added to the pre-ablation drug regimen. IP-positive patients were less likely to be treated with amiodarone (8/21 IP-positive patients vs. 12/14 IP-negative patients; $\mathrm{P}=0.01$ ) and were less likely to be treated with combination therapy (2/21 IP-positive patients compared to 8/14 IP-negative patients; $\mathrm{P}=0.002$ ).

Two of the IP-positive patients who became noninducible after ablation refused ICD implantation. All but 1 IP-positive patient who had no inducible VT after the ablation procedure $(n=15)$ remained free of VT during follow-up. This patient required a repeated ablation procedure. Only 1 of 14 IPnegative patients (7\%) remained free of VT during followup. This patient had been rendered noninducible at the end of the ablation procedure. 


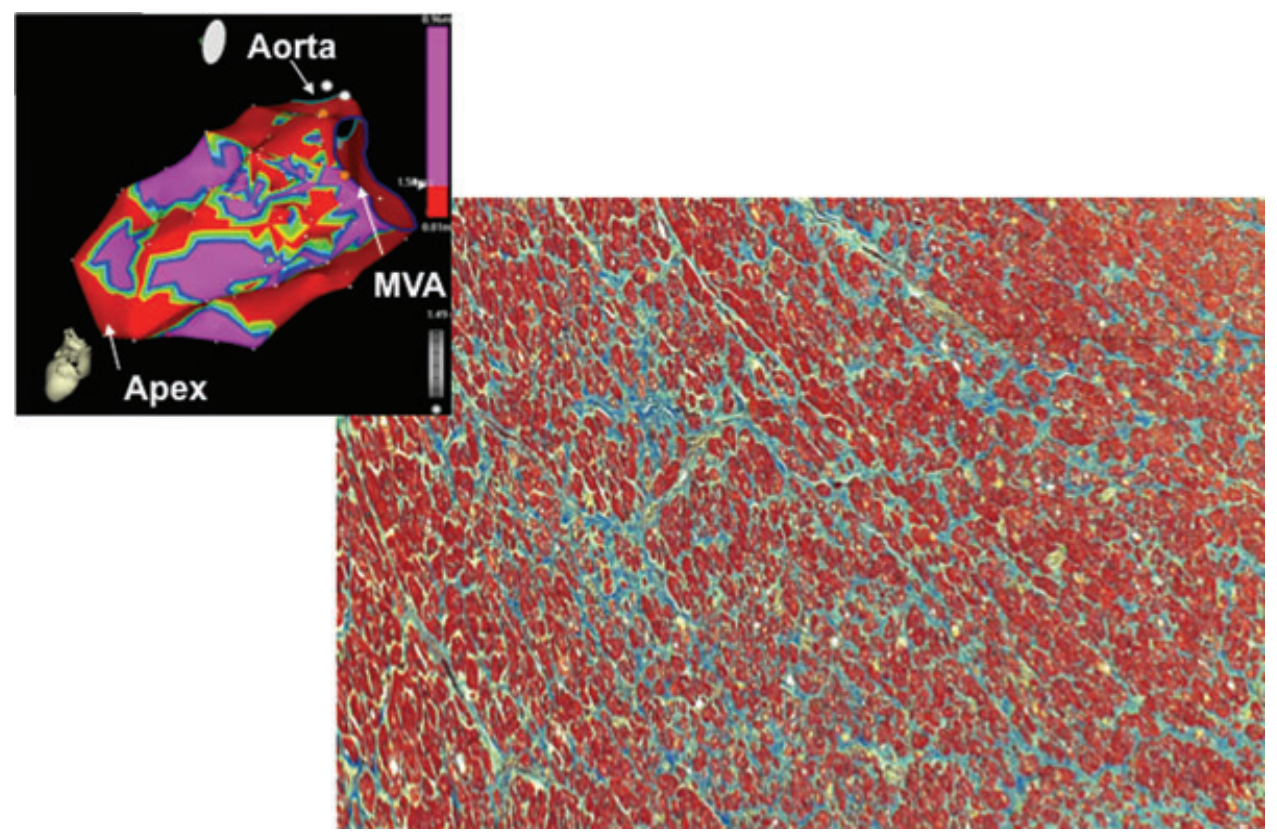

Figure 5. Left ventricular myocardium from the mid-lateral wall in a patient who underwent heart transplantation after a failed ablation procedure. There was no visible scarring at the endocardial site where the tissue was sampled. No IPs were found in this patient during endocardial and epicardial mapping. The tissue sample shows compact patches of fibrosis anastomosing and merging into more diffuse fibrosis. The sample is representative of the other sampled sites (Masson trichrome stain, 40×). An insert of the voltage map (top left) displays the endocardial voltage of this patient (low voltage [<1.5 $\mathrm{mV}$ ] is indicated in red color). Left ventricular apex, mitral valve annulus (MVA), and aorta are indicated.

The mean number of appropriate ICD therapies decreased from $47 \pm 72$ in the 3 months before the ablation procedure to $7 \pm 14$ during the first 3 months of follow-up $(\mathrm{P}=0.005)$ and to $14 \pm 27$ during a mean of $18 \pm 13$ months of followup $(\mathrm{P}=0.005)$. There was no significant difference in the number of ICD therapies in IP-positive patients compared to IP-negative patients in the 3 months before the ablation procedure $(P=0.1)$. ICD therapies after ablation decreased significantly in IP-positive patients but not in IP-negative patients (Table 4).

\section{Discussion}

\section{Main Findings}

This study demonstrates that IPs in patients with nonischemic cardiomyopathy identify critical sites for ablation of VT. IPs are not identifiable in all patients with nonischemic cardiomyopathy and the presence of IPs identifies a subgroup

\section{TABLE 4}

Prevalence of Appropriate Defibrillator Therapies Before and After the ICD Therapies in Patients With and Without Isolated Potentials

\begin{tabular}{lccc}
\hline ICD Therapies (n) & $\begin{array}{c}\text { IP-Positive } \\
(\mathbf{n}=\mathbf{2 1})\end{array}$ & $\begin{array}{c}\text { IP-Negative } \\
(\mathbf{n = 1 4})\end{array}$ & P-Value \\
\hline 3 months before ablation & $28 \pm 35$ & $70 \pm 97$ & 0.11 \\
3 months after ablation & $0.2 \pm 0.5^{*}$ & $16 \pm 18$ & 0.0007 \\
Total after ablation & $0.7 \pm 1.7 \dagger$ & $32 \pm 34$ & 0.0003 \\
\hline
\end{tabular}

${ }^{*} \mathrm{P}=0.007$ in IP-positive patients before ablation compared to 3 months postablation. $\dagger \mathrm{P}=0.007$ in IP-positive patients before ablation compared to the total follow-up period. ICD = implantable cardioverter defibrillator. with a favorable short- and mid-term prognosis after catheter ablation.

\section{Electrophysiologic Characteristics of Anatomic Substrate in IP-Positive Patients}

Patients with various types of nonischemic cardiomyopathy are described in this study, with the most common type being idiopathic dilated cardiomyopathy. Regardless of the type of cardiomyopathy, the presence of IPs appears to identify a subgroup of patients with a favorable prognosis. IPs were not present to the same extent in all forms of nonischemic cardiomyopathy. They were identified in all patients with ARVD and most patients with cardiac sarcoidosis but not in all patients with dilated cardiomyopathy. Although large areas of scar are uncommon in patients with nonischemic cardiomyopathy, extensive interstitial fibrosis is frequently seen histologically. ${ }^{4}$ Strands of fibrous tissue may serve as electrical barriers and result in electrogram fragmentation. The degree of fibrosis ${ }^{6}$ as well as the fiber orientation relative to the location of fibrotic barriers may be factors resulting in activation delay and electrogram fragmentation. ${ }^{7}$

In patients with idiopathic dilated cardiomyopathy, variable patterns of fibrosis have been described. ${ }^{6,7}$ It appears that some patients with nonischemic cardiomyopathy have a characteristic pattern of fibrosis that results in specific electrophysiologic properties. A corresponding electrogram pattern with multiple spikes separated by isoelectric segments has been described in patients with a high degree of fibrosis that has been characterized as replacement fibrosis, ${ }^{6}$ similar to postinfarction scars. As in postinfarction patients, critical areas often display IPs and the presence of IPs is a better marker for critical areas than low-voltage alone. These 
areas often are areas of slow conduction where block can occur. ${ }^{6,8}$ The presence of a fixed area of block indicates that the arrhythmogenic substrate is confined to a circumscribed area determined by the scar and therefore may be amenable to focal ablation lesions.

\section{Electrophysiologic Characteristics of the Anatomic Substrate in IP-Negative Patients}

In patients without IPs, although matching pace-maps were present, the outcomes after catheter ablation were less favorable than in patients in whom IPs were present. The diffuse pattern of fibrosis that was found histologically in 2 patients undergoing cardiac transplantation is similar to the pattern of moderate fibrosis described by Anderson et al. in some of patients with cardiomyopathy, in which the fibrosis did not interrupt myocyte fiber orientation. ${ }^{6}$ In these 2 patients, no IPs were identified despite extensive endocardial and epicardial mapping. It is possible that in the presence of moderate fibrosis, the exit site of a reentrant circuit may be less fixed and may more often be determined by functional lines of block.

\section{Prognostic Implications}

Similar to patients with ischemic cardiomyopathy, patients in whom no sites with IPs were identified during mapping of VT had a high probability of recurrent VT. ${ }^{3}$ Major complications associated with the failure to eliminate inducible VTs by ablation have been described previously in patients with nonischemic cardiomyopathy. ${ }^{9-11}$ It is possible that systematic epicardial mapping in all patients would have revealed IPs in some of the patients who did not have endocardial IPs. However, among the 7 patients with major arrhythmic events during follow-up, 3 patients had undergone percutaneous epicardial mapping and 1 had undergone limited epicardial mapping from the coronary venous system without detecting IPs. A possible explanation for the failure of catheter ablation is that the reentry circuits were less often anatomically fixed when IPs were absent.

\section{Prior Reports}

Soejima et al., ${ }^{12}$ Delacratez et al.., ${ }^{13}$ and Cano et al..$^{14}$ reported data on mapping and ablation in patients with nonischemic cardiomyopathy. The value of IPs however and possible prognostic implications were not assessed.

\section{Limitations}

A limitation of this study is that epicardial mapping was performed in only approximately one-third of patients who did not have endocardial IPs. A second limitation is that pacing from other ventricular locations, which may reveal late local activity that may not be recorded in sinus rhythm, was not performed in our study. Therefore, the prevalence of IPs in nonischemic cardiomyopathy may have been underestimated. Finally, histological samples were available only from IP-negative patients. Histological samples from IP-positive patients would have facilitated a comparison of the structural basis for the presence or absence of IPs.

\section{Conclusion}

IPs in conjunction with pace-mapping indicate critical areas of VT circuits in patients with nonischemic cardiomyopathy. Freedom from VT can be achieved by catheter ablation in most patients in whom IPs are detected during mapping of VT. Patients in whom IPs are present at critical ablation sites appear to have a better prognosis and fewer major adverse events than patients in whom the arrhythmogenic substrate is not characterized by IPs.

\section{References}

1. Bogun F, Bahu M, Knight B, Weiss R, Goyal R, Daoud E, Man K, Strickberger S, Morady F: Response to pacing at sites of isolated diastolic potentials during ventricular tachycardia in patients with previous myocardial infarction. J Am Coll Cardiol 1997;30:505-513.

2. Marchlinski FE, Callans DJ, Gottlieb CD, Zado E: Linear ablation lesions for control of unmappable ventricular tachycardia in patients with ischemic and nonischemic cardiomyopathy. Circulation 2000;101:1288-1296

3. Bogun F, Good E, Reich S, Elmouchi D, Igic P, Lemola K, Tschopp D, Oral H, Chugh A, Pelosi F, Morady F: Isolated potentials during sinus rhythm and pace-mapping within scars as guides for ablation of postinfarction ventricular tachycardia. J Am Coll Cardiol 2006;47:20132019.

4. de Bakker JM, van Capelle FJ, Janse MJ, Tasseron S, Vermeulen JT, de Jonge N, Lahpor JR: Fractionated electrograms in dilated cardiomyopathy: Origin and relation to abnormal conduction. J Am Coll Cardiol 1996;27:1071-1078.

5. Hummel D, Strickberger S, Daoud E, Niebauer M, Bakr O, Man K, Williamson B, Morady F: Results and efficiency of programmed ventricular stimulation with four extrastimuli compared with one, two, and three extrastimuli. Circulation 1994;90:2827-2823.

6. Anderson KP, Walker R, Urie P, Ershler PR, Lux RL, Karwandee SV: Myocardial electrical propagation in patients with idiopathic dilated cardiomyopathy. J Clin Invest 1993;92:122-140.

7. Kawara T, Derksen R, de Groot JR, Coronel R, Tasseron S, Linnenbank AC, Hauer RN, Kirkels H, Janse MJ, de Bakker JM: Activation delay after premature stimulation in chronically diseased human myocardium relates to the architecture of interstitial fibrosis. Circulation 2001;104:3069-3075

8. Pogwizd SM, McKenzie JP, Cain ME: Mechanisms underlying spontaneous and induced ventricular arrhythmias in patients with idiopathic dilated cardiomyopathy. Circulation 1998;98:2404-2414.

9. Koplan BA, Soejima K, Baughman K, Epstein LM, Stevenson WG: Refractory ventricular tachycardia secondary to cardiac sarcoid: Electrophysiologic characteristics, mapping, and ablation. Heart Rhythm 2006;3:924-929.

10. Haberl K, Allessie M: Differential effects of a segment of slow conduction on reentrant ventricular tachycardia in the rabbit heart. Circulation 1999;99:949-962

11. Hsia HH, Callans DJ, Marchlinski FE: Characterization of endocardial electrophysiological substrate in patients with nonischemic cardiomyopathy and monomorphic ventricular tachycardia. Circulation 2003;108:704-710.

12. Soejima K, Stevenson WG, Sapp JL, Selwyn AP, Couper G, Epstein LM: Endocardial and epicardial radiofrequency ablation of ventricular tachycardia associated with dilated cardiomyopathy: The importance of low-voltage scars. J Am Coll Cardiol 2004;43:1834-1842.

13. Delacretaz E, Stevenson WG, Ellison KE, Maisel WH, Friedman PL: Mapping and radiofrequency catheter ablation of the three types of sustained monomorphic ventricular tachycardia in nonischemic heart disease. J Cardiovasc Electrophysiol 2000;11:11-17.

14. Cano O, Hutchinson M, Lin D, Garcia F, Zado E, Bala R, Riley M, Cooper J, Dixit S, Gerstenfeld E, Callans D, Marchlinski FE: Electroanatomic substrate and ablation outcome for suspected epicardial ventricular tachycardia in left ventricular nonischemic cardiomyopathy. J Am Coll Cardiol 2009;54:799-808. 